1999

\title{
Noise Driven Avalanche Behavior in Subexcitable Media
}

Jichang Wang

Sándor Kádár

PeterJung

Kenneth Showalter

Follow this and additional works at: https://researchrepository.wvu.edu/faculty_publications

\section{Digital Commons Citation}

Wang, Jichang; Kádár, Sándor; Jung, Peter; and Showalter, Kenneth, "Noise Driven Avalanche Behavior in Subexcitable Media" (1999). Faculty Scholarship. 245.

https://researchrepository.wvu.edu/faculty_publications/245 


\title{
Noise Driven Avalanche Behavior in Subexcitable Media
}

\author{
Jichang Wang, ${ }^{1}$ Sándor Kádár, ${ }^{1}$ Peter Jung, ${ }^{2}$ and Kenneth Showalter ${ }^{1, *}$ \\ ${ }^{1}$ Department of Chemistry, West Virginia University, Morgantown, West Virginia 26506-6045 \\ ${ }^{2}$ Department of Physics and Astronomy, Ohio University, Athens, Ohio 45701
}

(Received 19 October 1998)

\begin{abstract}
The interaction of noise with a subexcitable chemical system gives rise to spatiotemporal behavior with novel statistical properties. Wave behavior in a photosensitive Belousov-Zhabotinsky reaction with imposed noise is monitored and statistically analyzed. Waves are initiated at sites where the accumulation of subthreshold perturbations induces local excitations, and waves propagate through the medium by a facilitated percolation process. Statistical analysis of the coherent spatiotemporal structures reveals power-law relations that indicate the system has no intrinsic length or time scale. [S0031-9007(98)08304-5]

PACS numbers: 82.40.Bj, 05.40.Ca, 05.45.Jn, 05.65.+b
\end{abstract}

Noise is usually thought to have a disruptive influence on spatial structures in dynamical systems: Stationary patterns tend to become "fuzzy" and traveling waves may break up or even fail to propagate. It is now known, however, that noise may give rise to ordered behavior and even new dynamical states [1-5], including noise supported pulses in diode arrays [6], and noise induced coherence in arrays of coupled dynamical elements $[7,8]$. Recent theoretical studies $[9,10]$ and experiments with cultured glial cells [11] and the Belousov-Zhabotinsky (BZ) reaction $[12,13]$ have shown that noise may play a constructive role in supporting spatiotemporal wave behavior [14], similar to the noise enhanced signal detection seen in stochastic resonance $[15,16]$. In this Letter, we describe noise initiated and sustained wave behavior in the BZ reaction and offer a statistical analysis of the corresponding coherent spatiotemporal structures [17]. We find power-law relations in the noisy BZ system as well as in a generic model for excitable media that are characteristic of avalanche behavior and indicative of no inherent length or time scale $[18,19]$.

Experiments were carried out with ruthenium(II)bipyridyl, a light sensitive catalyst of the BZ reaction, immobilized in a thin slab of silica gel. The gel was cast onto a microscope slide and placed into a reactor that was continuously fed with fresh, catalyst-free BZ solution to maintain constant, nonequilibrium conditions [20]. A $12 \mathrm{~mm} \times 12 \mathrm{~mm}$ image produced by a computer-controlled video projector $[14,21]$ was projected onto the face of the gel medium. Bromide ion is produced in a photochemical cycle when the ruthenium catalyzed BZ reaction is exposed to $460 \mathrm{~nm}$ light, which inhibits the autocatalysis of the reaction [22]. The excitability of the system can therefore be adjusted by varying the light intensity to a reference level that maintains the medium slightly below the excitability threshold. The illumination field was divided into an array of square cells, with the light intensity in each cell changed at equal-time intervals to random values above or below the reference level, which were
Gaussian distributed with a mean value equal to the reference level.

An example of the noise mediated initiation and evolution of wave behavior is illustrated in Fig. 1, where panel (A) shows the medium in the absence of noise, and panel (F) shows a typical imposed noise pattern. In the absence of noise, no wave initiations occur and any existing waves are quickly extinguished in the subexcitable medium. When noise is applied, waves are randomly initiated in cells where the accumulated perturbations cause the system to become excited. The wave initiated in panel (B) expands and is subsequently broken to form a spiral segment, as shown in panel (C). A successful initiation gives rise to an increasing level of wave activity, as shown in panels (D) and (E), since the noise both supports propagation and induces wave breaks. Cells in front of an advancing wave are excited if their recent noise history is biased toward the excitable state; cells with a noise history biased toward lower excitability cause wave breaks, giving rise to a spawning of embryo wave fragments. Hence, once wave nucleation occurs, there is a proliferation of wave activity throughout the medium, with the system evolving to support a large number of wave segments on wide space and time scales.

The noise mediated wave behavior can be simulated with the Barkley model [23], a computationally efficient, generic scheme for excitable media, which we modify to account for the influence of spatiotemporal noise. With the parameters chosen so that the medium is subexcitable, the noise is introduced by varying a parameter representing light intensity to random values above and below the reference level in each cell of the noise grid. The positive perturbations correspond to less light, which tends to induce excitation, while the negative perturbations correspond to more light, which tends to suppress wave activity. Figure 2 shows the noise mediated wave behavior in the model system, with the noise-free subexcitable state in panel (A) and a typical noise pattern in panel (F). Panel (B) shows a wave initiation and the subsequent evolution of the wave is shown in panel (C). Panels (D) and 

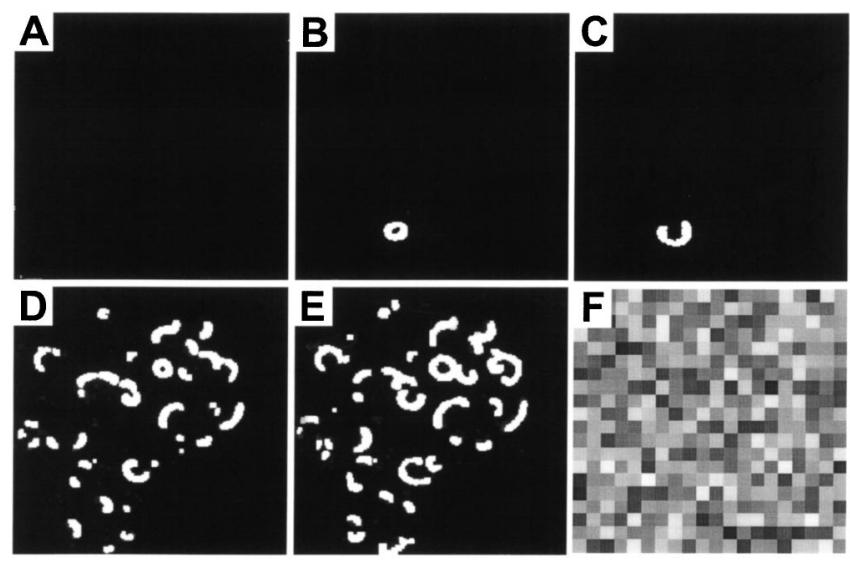

FIG. 1. Images showing wave behavior in noisy subexcitable BZ medium. Noise-free autonomous system at reference intensity $I_{0}=120(\mathrm{~A})$; successive frames at $5 \mathrm{~s}$ intervals showing wave initiation with imposed noise $[(\mathrm{B}),(\mathrm{C})]$; successive frames at $5 \mathrm{~s}$ intervals showing noise mediated wave propagation and fragmentation, as well as a new wave initiation [(D), (E)]; typical noise pattern $(\mathrm{F})$. The gray level in panels $(\mathrm{A})-(\mathrm{E})$ is proportional to the concentration of the catalyst $\mathrm{Ru}(\mathrm{bpy})_{3}^{3+}$, with white and black representing high and low values, respectively. The region of imposed noise in the illumination field consists of a 20 cell $\times 20$ cell square array, with a total area of $12 \mathrm{~mm} \times 12 \mathrm{~mm}$. The light intensity in each cell is adjusted at $10 \mathrm{~s}$ intervals to random values according to $I=I_{0}\left[1+\frac{1}{2} G(x, \delta)\right]$; with $|x| \leq 2 \delta$, where $I_{0}$ is the reference intensity, and $G(x, \delta)$ is a Gaussian distributed random number with a standard deviation of $\delta=0.8$. The reaction was carried out at $22.0^{\circ} \mathrm{C}$ with stock solutions maintained at $0{ }^{\circ} \mathrm{C}$ to prevent decomposition. Composition of the catalyst-free BZ reaction mixture: $0.28 \mathrm{M} \mathrm{NaBrO}, 0.025 \mathrm{M}$ malonic acid, $0.165 \mathrm{M}$ bromomalonic acid, $0.4 \mathrm{M} \mathrm{H}_{2} \mathrm{SO}_{4}$. The silica gel medium was prepared by acidifying a solution of $10 \%$ (w/w) $\mathrm{Na}_{2} \mathrm{SiO}_{3}$ and $2.0 \mathrm{mM} \mathrm{Ru}(\mathrm{bpy})_{3}^{2+}$ with $\mathrm{H}_{2} \mathrm{SO}_{4}$ and casting a uniform $0.3 \mathrm{~mm} \times 20 \mathrm{~mm} \times 30 \mathrm{~mm}$ layer onto a microscope slide. The intensity of the projected image was adjusted at each pixel by an iterative algorithm to ensure spatial homogeneity of the illumination field [21]. The light was passed through a $460 \mathrm{~nm}$ bandpass filter, and the intensity was varied on an eight-bit gray scale between 0 and 255 . The noise pattern was updated at $10 \mathrm{~s}$ intervals and the wave behavior was monitored every $5 \mathrm{~s}$ during a noise-free period of $50 \mathrm{~ms}$. The maximum and minimum irradiance of the projected image, measured at the surface of the gel medium, was 5.7 and $0.1 \mathrm{~mW} \mathrm{~cm}^{-2}$, with the reference level between these extremes at $2.9 \mathrm{~mW} \mathrm{~cm}^{-2}$.

(E) show fully developed wave activity with noise induced wave breaks and initiations.

The imposed noise gives rise to two distinct interactions with the reaction-diffusion system: (1) Wave initiations occur from the local accumulation of favorable noise perturbations, and (2) noise both supports wave propagation and induces wave breaks. Noise induced wave initiation is a nucleation process with features akin to self-organized criticality $[18,19]$. The subexcitable medium does not respond with an excitation until there is a sufficient local accumulation of perturbations, with the positive perturbations outweighing the negative ones. This accumula-
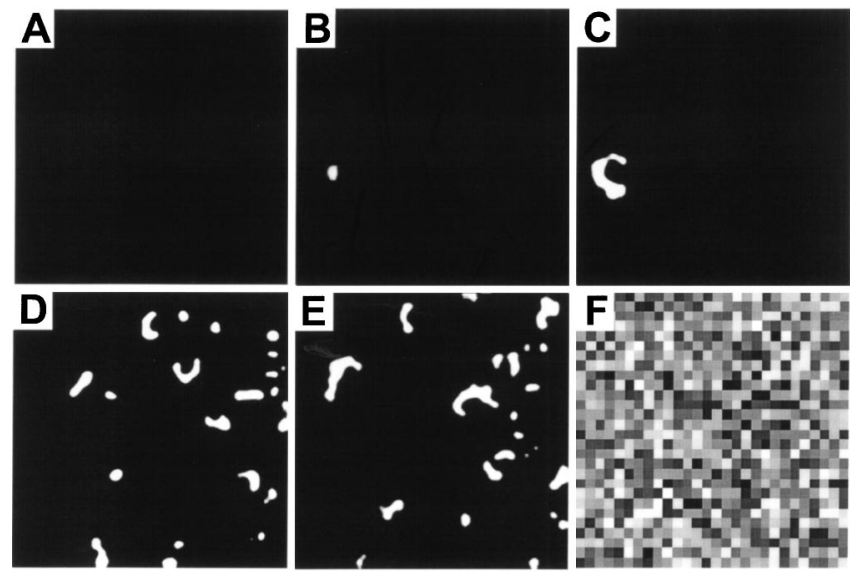

FIG. 2. Images showing wave behavior generated from the numerical integration of the modified Barkley model [23]. Noise-free autonomous system at reference level $\phi_{0}=0.0288$ (A); successive frames at intervals of $200 \Delta t$ showing wave initiation with imposed noise $[(\mathrm{B}),(\mathrm{C})]$; successive frames at intervals of $350 \Delta t$ showing wave propagation, fragmentation, and initiation with imposed noise [(D),(E)]; typical noise pattern (F). The model is given by $\partial u / \partial \tau=D_{u} \nabla^{2} u+$ $f[u, v, \phi(\tau)], \partial v / \partial \tau=g(u, v)$, where the functions $f$ and $g$ are defined as $f=(1 / \epsilon) u(1-u)[u-(v+b+\phi) / a]$ and $g=u-v$. The variables $u$ and $v$ are the activator and inhibitor species, $\nabla^{2}$ is the Laplacian operator, $D_{u}$ is the diffusion coefficient of $u$, and $\phi(\tau)$ is a time dependent parameter determining the local excitability. The simulation was carried out using a two-dimensional lattice of $280 \times 280$ grid points with the Laplacian operator $\nabla^{2}$ approximated by a standard five-point formula $(\Delta x=0.0111, \Delta t=0.003084)$. The gray level [given by $\operatorname{int}\left(u / u_{\max } \times 255\right)$ ] represents the concentration of the activator species. The cell size in the noise pattern is $10 \times 10$ grid points, and the noise refresh interval is $50 \Delta t$. The parameter $\phi$ was adjusted in each cell at equal time intervals according to $\phi=\phi_{0}\left[1+\frac{1}{2} G(x, \delta)\right]$ with $|x| \leq 2 \delta$, where $\phi_{0}$ is the reference intensity, and $G(x, \delta)$ is a Gaussian distributed random number with a standard deviation of $\delta=$ 1.0. The behavior is oscillatory for $\phi=0$, but increasing this parameter drives the system to the excitable state and, eventually, to the subexcitable regime, where sustained wave propagation is not supported by the medium. The parameter values $\phi_{0}=0.0288, a=0.5, b=-0.15$, and $\epsilon=0.005$ were chosen to model behavior in the subexcitable regime.

tion can be readily verified in the numerical simulations; the initiations in Fig. 2, for example, occurred only after many noise perturbations. Figure 3 shows how the wave initiation in panel (B) of Fig. 2 required five positive perturbations to induce an initial excitation, which was subsequently suppressed by a negative perturbation but was then revived by a positive perturbation. Even though a single positive perturbation cannot excite a cell, multiple positive perturbations, even when interspersed with negative perturbations, are able to give rise to an excitation due to an accumulative effect on the medium. The noise and chemical reaction time scales play a fundamental role in this behavior; in these experiments and simulations the noise period was always on a shorter time scale than the response time of the chemical reaction. 


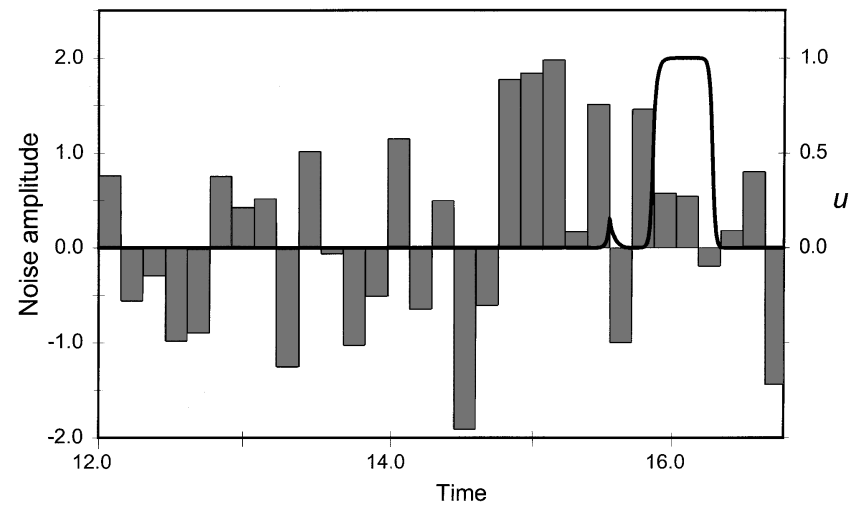

FIG. 3. Wave initiation from accumulation of perturbations. Bars show positive and negative perturbations at initiation site in Fig. 2(B); solid curve shows time series of the activator species $u$ at that site responding to the noise perturbations. Full excitation with $u=1.0$ corresponds to wave initiation; $u$ subsequently decreases in refractory wave back as wave propagates outward. Parameters and conditions of calculation are the same as in Fig. 2.

The second mode of behavior, noise supported wave propagation, differs from wave nucleation in that the wave provides autocatalyst for initiating nearby sites. Once a wave is initiated, it advances into advantageous sitescells with noise histories that make them susceptible to excitation. Thus, the propagation is akin to percolation [24-26]; however, it is dependent on both the local noise history and the diffusion of autocatalyst from the nearby wave front. In contrast to percolation, the probability for a cell to be in the excited state is typically much less than 0.5 , and the excited regions take on the form of curved wave segments rather than the geometry of the noise grid.

The statistical properties of the spatiotemporal behavior can be analyzed by cataloging the coherent structures according to size and subsequently determining the probability of each size occurring. The time dependence of each structure is taken into account by stacking $N$ images of the medium at equal time intervals, $\Delta t$, to construct a space-time cube that carries all of the spatiotemporal information within the time interval $N \Delta t$. The space-time volume of each coherent structure is then determined by identifying all of the grid sites where the reaction is in the excited state and defining each of these by a small cube, for example, 2 pixels $\times 2$ pixels $\times \Delta t$. The spatial side lengths of these cubes are chosen such that neighboring cubes touch each other to form the area of the coherent structure at time $t_{n}$. The temporal side length is chosen such that excited cubes at successive times $t_{n}$ and $t_{n+1}$ touch each other to form the space-time volume of the coherent structure. We consider connected cubes only in the time-forward direction as belonging to one cluster in order to avoid counting two colliding clusters with uncorrelated origins as one coherent structure. When two waves collide to form one new wave, the coherent cluster corresponding to one of the incoming waves

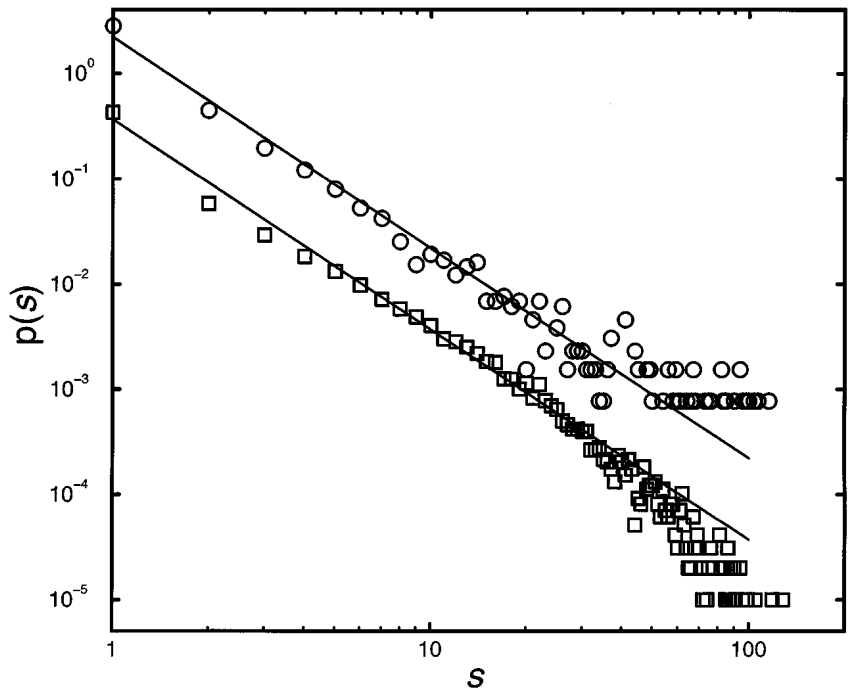

FIG. 4. Coherent structure probability density $p(s)$ as a function of structure size $s$. Distribution from experimental measurements $(\bigcirc)$ and from model calculations $(\square)$ generated from images like those in Figs. 1 and 2 . The probability density $p(s)$ is obtained by dividing the number of clusters whose size falls within the interval $s \pm \Delta s$ by the total number of clusters $N: p(s)=N_{s} / N$, where $s$ is 1 pixel $\times 1$ pixel $\times 5 \mathrm{~s}$ in the experiments and 1 pixel $\times 1$ pixel $\times 50 \Delta t$ in the calculations. The cluster size $s$ is normalized by the size interval $2 \Delta s$, where $\Delta s$ is 5 in the experiments and 100 in the calculations. The cluster size normalization does not affect the slope of the distribution curves, indicating a statistical scale invariance of the system. The solid lines are given by the power law $p(s)=b s^{a}$, where $s$ is cluster size, $b$ is a constant, and $a$ is the exponent set equal to 2.0. The experimental $p(s)$ values were multiplied by a factor of 4.0 to allow their visualization, since the values overlap with the model calculation $p(s)$ values.

is terminated at the collision. The overall time interval $N \Delta t$ is chosen such that a sufficiently large number of coherent structures form with statistically distributed sizes.

The occurrence of a coherent structure of size $s$ is characterized by the cluster size distribution function $p(s)$, as shown in Fig. 4. The experimental measurements as well as the simulations with the Barkley model yield powerlaw distributions as a function of cluster size, each with an exponent close to 2.0. Little change in the exponent was found on varying the noise length and time scales. The scatter in the data at large values of $s$ in the experimental plot arises from a limited number of measurements due to the practical constraints of the experiment. Less scatter is exhibited in the model calculations, since a large number of values could be obtained. The power-law relation for the size distribution suggests that the wave behavior is characterized by no intrinsic length or time scale. The noise pattern continually changes from one randomly determined pattern to the next, which, in principal, allows the coherent space-time structure to extend unbounded in the time coordinate. This is also true for the space coordinates; however, the probability of all cells having such a favorable history is small. 
Noise mediated wave behavior in the BZ reaction and the Barkley model provides insights into related noise supported waves in biological systems. Kainate induced calcium waves in networks of cultured glial cells [11] display features much like those reported here, with wave activity appearing only above a threshold noise level and noise induced wave breaks appearing when the noise level is increased further. Remarkably, this system also exhibits power-law behavior with a similar critical exponent. The noise mediated waves and power-law behavior in these networks suggest a possible mechanism for long-range signaling and memory in neuronal tissues.

We thank the National Science Foundation (CHE9531515) and the Office of Naval Research for supporting this research.

*To whom correspondence should be addressed.

[1] R. Kapral and W.-G. Wu, in Chemical Waves and Patterns, edited by R. Kapral and K. Showalter (Kluwer, Dordrecht, The Netherlands, 1995), pp. 609-634.

[2] A. J. Irwin, S. J. Fraser, and R. Kapral, Phys. Rev. Lett. 64, 2343-2346 (1990).

[3] J. M. R. Parrondo, C. Van Den Broeck, J. Buceta, and F. J. De La Rubia, Physica (Amsterdam) 224A, 153-161 (1996).

[4] J. M. G. Vilar and J. M. Rubí, Phys. Rev. Lett. 78, 28862889 (1997).

[5] A. S. Mikhailov, Phys. Rep. 184, 307-374 (1989).

[6] M. Löcher, D. Cigna, and E. R. Hunt, Phys. Rev. Lett. 80, 5212-5215 (1998).

[7] J. F. Linder, B. K. Meadows, W. L. Ditto, M. E. Inchiosa, and A. R. Bulsara, Phys. Rev. Lett. 75, 3-6 (1995).
[8] M. Löcher, G. A. Johnson, and E. R. Hunt, Phys. Rev. Lett. 77, 4698-4701 (1996).

[9] P. Jung and G. Mayer-Kress, Phys. Rev. Lett. 74, 21302133 (1995).

[10] P. Jung and G. Mayer-Kress, Chaos 5, 458-462 (1995).

[11] P. Jung, A. Cornell-Bell, K. S. Madden, and F. Moss, J. Neurophysiol. 79, 1098-1101 (1998).

[12] A. N. Zaikin and A.M. Zhabotinsky, Nature (London) 225, 535-537 (1970).

[13] A. T. Winfree, Science 175, 634-636 (1972).

[14] S. Kádár, J. Wang, and K. Showalter, Nature (London) 391, 770-772 (1998).

[15] K. Wiesenfeld and F. Moss, Nature (London) 373, 33-36 (1995).

[16] L. Gammaitoni, P. Hanggi, P. Jung, and F. Marchesoni, Rev. Mod. Phys. 70, 223-287 (1998).

[17] P. Jung, Phys. Rev. Lett. 78, 1723-1726 (1997).

[18] P. Bak, C. Tang, and K. Wiesenfeld, Phys. Rev. Lett. 59, $381-384$ (1987).

[19] P. Bak, K. Chen, and M. Creutz, Nature (London) 342, 780-782 (1989).

[20] Z. Noszticzius, W. Horsthemke, W. D. McCormick, H. L. Swinney, and W. Y. Tam, Nature (London) 329, 619-620 (1987).

[21] V. Petrov, Q. Ouyang, and H. L. Swinney, Nature (London) 388, 655-657 (1997).

[22] S. Kádár, T. Amemiya, and K. Showalter, J. Phys. Chem. 101, 8200-8206 (1997).

[23] D. Barkley, Physica (Amsterdam) 49D, 61-70 (1991).

[24] D. Stauffer, Introduction to Percolation Theory (Taylor and Francis, London, 1992), 2nd ed.

[25] M. Sahimi, AIChE. J. 41, 229-240 (1995).

[26] I. Sendiña-Nadal, D. Roncaglia, D. Vives, V. PérezMuñuzuri, M. Gómez-Gesteira, V. Pérez-Villar, J. Echave, J. Casademunt, L. Ramírez-Piscina, and F. Sagués, Phys. Rev. E 58, R1183-R1186 (1998). 